\title{
REBOA in Canada: time to shine, or time's up?
}

\author{
Winny $\mathrm{Li}^{1} \cdot$ Andrew Petrosoniak ${ }^{1,2}$ (D) Markus Ziesmann ${ }^{3}$ \\ Received: 1 November 2020 / Accepted: 28 November 2020 \\ (c) Canadian Association of Emergency Physicians (CAEP)/ Association Canadienne de Médecine d'Urgence (ACMU) 2020
}

Keywords Trauma $\cdot$ REBOA $\cdot$ Technology

Every day we are sold new ideas, new designs, and new technologies, often with a marketing pitch that they will improve our lives in some capacity. The new, however, often fails to entirely replace the old. Trauma care is no different. Innovations emerge, championed by enthusiastic early adopters, followed by gradual evolution of evidence informing use and adoption-and sometimes dampening early enthusiasm. Resuscitative endovascular balloon occlusion of the aorta (REBOA), a technique originally used during the Korean war for managing non-compressible hemorrhage is trauma's "newest" technology [1]. REBOA has been implemented at multiple US trauma centers despite lacking prospective evidence [1]. Champions point to retrospective data suggesting REBOA leads to improved survival in certain cohorts [2] while critics note no differences in transfusion, hospital length of stay or overall survival benefit $[3,4]$ while conferring increased morbidity such as acute kidney injury, and lower limb amputations [3].

Treating non-compressible hemorrhage remains an incredible clinical challenge. As we learn more about REBOA, there is a need for a concerted effort to define and identify which patients REBOA will most likely benefit. Without timely access to definitive surgical care, functional massive transfusion protocols, support of vascular surgeons and interventional radiologists, and longitudinal financial, educational, and quality improvement resources, a REBOA program simply cannot be implemented. In lower volume

Andrew Petrosoniak

petro82@gmail.com

1 Division of Emergency Medicine, Department of Medicine, St. Michael's Hospital, University of Toronto, 30 Bond Street, Toronto M5B 1W8, ON, Canada

2 St. Michael's Hospital, University of Toronto, Toronto, ON, Canada

3 Department of Surgery, University of Manitoba, Winnipeg, MB, Canada centers-like most Canadian trauma centers - a refined patient selection process will paramount to justify the cost and implementation efforts $[5,6]$.

\section{A Canadian REBOA gap analysis}

The study by Eksteen and colleagues is the first to evaluate the role of REBOA in a Canadian context [7]. The authors conducted a retrospective gap analysis of 3415 trauma-registry patients who presented to two Level 1 trauma centers in Edmonton, Alberta [7]. Using clearly defined selection criteria, $1.1 \%(n=38)$ of the study population met eligibility criteria for REBOA deployment. The authors conclude that a "small but significant number of trauma patients at the two trauma centers were identified as potential candidates for REBOA use". The precise estimate of $1.1 \%$ eligibility may not describe the same eligibility in all hospital environments. The cohort reflects the selection-bias of those triaged to tertiary-care trauma centers, indicating a higher injury severity than an entire trauma cohort. Additionally, several patients were excluded based on information available only retrospectively, when REBOA might have been initially indicated and under-estimating the REBOA application rate. Despite some modest limitations, this data provides us with a more detailed understanding of the opportunity for REBOA in Canada and to our knowledge reflects the best available data of its kind.

While the application of REBOA is frequently described in blunt trauma, it is interesting to note that only $10 \%$ of this study population sustained penetrating injury, but this cohort accounted for $32 \%$ of REBOA-eligible patients. Clinicians looking to implement their own REBOA programs must not underestimate this cohort of patients [8]. 


\section{REBOA in Canada: a small part of a larger system}

So what now? Is Canada ready for widespread REBOA implementation? This demands extensive resources for a small number of potential patients. Eksteen and colleagues found a $1.1 \%$ eligibility, but we cannot conclude that this cohort truly would benefit from REBOA deployment. The investment returns of trauma-program changes must be examined at a local level, to identify opportunities for maximal impact. Based on this data, most Canadian centers would be low-volume users of REBOA [9]. The Edmonton experience of 38 eligible cases at two hospitals over 3 years would result in approximately six REBOA deployments per site per year. The value of experience and practice cannot be understated. REBOA success increases in high and mid-volume REBOA hospitals compared to low, with decreased odds of mortality [9]. Canadian cities with multiple trauma centers may consider triaging REBOA-eligible cases to one site to maximize singlecenter experience, however this remains challenging as pre-hospital selection may not be feasible. REBOA application has substantive trauma system implications that expand beyond the walls of a single institution.

This excellent study by Eksteen and colleagues highlights more than just the REBOA gap analysis; the authors identified several unintended outcomes of their study, namely, quality gaps in their trauma program. They have now undertaken quality improvement initiatives to optimize existing processes. REBOA is not a panacea; it is critical that a REBOA program be part of a larger, comprehensive damage control resuscitation strategy that combines rapid access to blood products with definitive hemorrhage control. In some centers, we might find that efforts to improve the core elements of trauma care may improve patient outcomes and obviate the need for interventions like REBOA.

\section{Where do we go from here?}

Most Canadian emergency departments will never be considered "high-volume" REBOA centers, so the implementation strategy must be even more robust to offset its infrequent use. Based on the literature and the contribution by Eksteen and colleagues, several principles should be considered:

1. A REBOA program requires multi-disciplinary leadership including representatives from emergency medicine, trauma surgery, vascular surgery, nursing and posttrauma care specialists.
2. The trauma program undertakes an in-depth review to ensure key performance targets are being met (e.g. time to blood administration) to ensure care protocols are optimized.

3. Regular, team-based and operator skills training to ensure the necessary technical skill sets are achieved and maintained.

4. A robust quality assurance process to regularly conduct audits of trauma care of both patients who do and do not receive REBOA.

Currently the evidence for REBOA is ambiguous, with heterogeneous patient cohorts not-quite representing our own, who are cared for in trauma systems different than our own, and with injury patterns different from those we see. There are identifiable patients deriving both benefit and harm from its implementation. Moving forward, we must refine our questions from "does REBOA work" to "does REBOA work for specific conditions in my patients". Eksteen and colleagues contribute to answering this question and we encourage further work in a Canadian context. For now, it remains to be seen what the enduring legacy of REBOA will be: timeless classic, or historical anachronism.

\section{Compliance with ethical standards}

Conflict of interest The authors declare that they have no conflict of interests.

\section{References}

1. Bulger EM, Perina DG, Qasim Z, Beldowicz B, Brenner M, Guyette F, et al. Clinical use of resuscitative endovascular balloon occlusion of the aorta (REBOA) in civilian trauma systems in the USA, 2019: a joint statement from the American College of Surgeons Committee on Trauma, the American College of Emergency Physicians, the National Association of Emergency Medical Services Physicians and the National Association of Emergency Medical Technicians. Trauma Surg Acute Care Open. 2019;4(1):e000376-e386.

2. Brenner M, Moore L, DuBose J, Scalea T. Resuscitative endovascular balloon occlusion of the aorta (REBOA) for use in temporizing intra-abdominal and pelvic hemorrhage: physiologic sequelae and considerations. Shock. 2020;54(5):615-22.

3. Joseph B, Zeeshan M, Sakran JV, Hamidi M, Kulvatunyou N, Khan M, et al. Nationwide analysis of resuscitative endovascular balloon occlusion of the aorta in civilian trauma. JAMA Surg. 2019;154(6):500-8.

4. DuBose JJ, Scalea TM, Brenner M, Skiada D, Inaba K, Cannon $\mathrm{J}$, et al. The AAST prospective aortic occlusion for resuscitation in trauma and acute care surgery (AORTA) registry: data on contemporary utilization and outcomes of aortic occlusion and resuscitative balloon occlusion of the aorta (REBOA). J Trauma Acute Care Surg. 2016;81(3):409-19.

5. Ziesmann MT, Rezende-Neto J, McKendy K, Prabhudesai V, Rizoli S, Petrosoniak A. In the zone: lessons from the first Canadian emergency department application of resuscitative 
endovascular balloon occlusion of the aorta (REBOA). CJEM. 2019;21(3):430-4.

6. Paradis T, Bekdache O, Bracco D, Grushka J, Razek T, Lasry D, et al. Deployment of second-generation resuscitative endovascular balloon occlusion of the aorta for unresponsive hypotension in a polytrauma patient. Can J Surg. 2019;62(2):142-4.

7. Eksteen A, Domhnall O, Odenbach J, Douma MJ, O'Neill K, Anantha R, Bradley N, Gauri A, Widdler S. A gap analysis of the potential use of resuscitative endovascular balloon occlusion of the aorta (REBOA) in trauma at two major Canadian trauma centers. CJEM. 2020. https://doi.org/10.1007/s43678-020-00007 -5 .
8. Barnard EBG, Morrison JJ, Madureira RM, Lendrum R, FragosoIñiguez $\mathrm{M}$, Edwards $\mathrm{A}$, et al. Resuscitative endovascular balloon occlusion of the aorta (REBOA): a population based gap analysis of trauma patients in England and Wales. Emerg Med J. 2015;32(12):926-32.

9. Theodorou CM, Anderson JE, Brenner M, Scalea TM, Inaba $\mathrm{K}$, Cannon J, et al. Practice, practice, practice! effect of resuscitative endovascular balloon occlusion of the aorta volume on outcomes: data from the AAST AORTA registry. J Surg Res. 2020;253:18-25. 